\title{
THE UNIVERSITY OF MISSQURI
}

\section{ENGINEERING REPRINT SERIES}

Reprint Number 82

Engineering Experiment Station

\section{METHODS OF MEASUIRING}

\section{ELECTRIC FIELDS \\ Byron W. Sherman \\ ine}

Assissant Professor of Electrical Engineering

D) University of Missouri

Columbia. Missouri

Donald L. Waidelich

Professor of Electrical Engineering

University of Missouri

Reprinted from

Supplement to EEE Transactions on Aerospace and

Electronic Systems

Vol. AES-2, No. 6 November 1966 


\section{COLLEGE OF ENGINEERING \\ THE ENGINEERING EXPERIMENT STATION}

The Engineering Experiment Station was organized in 1909 as a part of the College of Engineering. The staff of the Station includes all members of the Faculty of the College of Engineering, together with Research Assistants supported by the Station Funds.

The Station is primarily an engineering research institution engaged in the investigation of fundamental engineering problems of general interest, in the improvement of engineering design, and in the development of new industrial processes.

The Station desires particularly to co-operate with industries of Missouri in the solution of such problems. For this purpose, there is available not only the special equipment belonging to the Station but all of the equipment and facilities of the College of Engineering not in immediate use for class instruction.

Inquiries regarding these matters should be addressed to:

The Director

Engineering Experiment Station

University of Missouri

Columbia, Missouri 
by B. W. Sherman and D. L. Waidelich University of Missouri

\section{ABSTRACT}

To determine the potential of a spacecraft, an electric field meter is needed to measure the field at the surface and in the neighborhood of the craft. A search was made for field measuring methods which would not involve rapidly moving parts. Several of these methods are discussed along with some experimental work performed to determine their feasibility. The use of the stark effect in the rotational spectra of gases appears to be the most promising method considered and it is discussed in more detail.

\section{INTRODUCTION}

In determining the potential of a spacecraft, an electric field meter is needed to measure the three components or the magnitude and direction of the electric field at the surface and in the neighborhood of the craft. The first work done in the measurement of the electric field was at the surface of the earth, and later measurements were made by the use of balloons and airplanes ${ }^{1}$. Instruments using antennas or probes were employed to pick up a charge and then were discharged through a measuring instrument. The time for the conductors to attain their final potential was long, and so point discharges, water drops, flames, $\mathrm{X}$-ray and ultraviolet sources, and radioactive sources were used to increase the conductivity of the medium and thereby accelerate the process?.

Another instrument for measuring the electric field is the field mill 3 which consists of a rotating plate adjacent to a stationary plate. The rotating plate alternately covers and uncovers the stationary plate and thus produces an alternating voltage which may be amplified and measured. Some field mill measurements have been made on rockets and satellites 4 but difficulty has been encountered because the plasma sheath about the instrument shields it from the electric field to be measured 5 . The vibrating charged probe 6 is another method that has been used on the Gemini satellites to measure the electric field intensity. Still another method is the use of the double probe 7 in which the spacing of the probes should be great enough so that the two sheaths do not overlap and thus

This work was supported in part by the National Science Foundation and the National Aeronautics and Space Administration. shield the probes from the electric field to be measured.

Both the fleld mill and vibrating types of meters involve motion which may cause trouble for a spacecraft on a long mission in space. The probe methods usually require a large distance between the probes and have difficulties when the potential difference associated with the electric field is the same order of magnitude as the potential drop across the sheath.

A search was made for methods of meas uring electric fields that would not involve rapidly moving parts. The first method considered was that of measuring the force on a charge in an electric field. Calculations and a few simple experiments involving charged dielectric sheets and electrets quickly demonstrated that the forces involved would be too small for measurement of the expected small fields. For relatively large fields of the order of 50 volts per centimeter, this method might be quite use ful.

Ferroelectric materials such as barium titanate were next investigated with the view of using one of them as the dielectric of a capacitor. The capacitance was to be measured by the use of a bridge circuit or by the change in the frequency of an oscillator. Unfortunately, the conductance of the material was so high that only transient effects could be noticed. Another possible method was to use ferroelectric materials in a manner similar to the ferromagnetic materials used in a flux-gate magnetometer 8 . Again the conductance of the materials presented a difficulty, but the major problem was that the ferroelectric material would not saturate well enough.

The next method investigated was the use of the Stark effect in the rotational spectra of gases, and this appears to be the most promising of the methods considered. Several factors made the Stark effect appear attractive as a means of measuring static fields in space. The similar technique of measuring static magnetic fields by means of the Zeeman effect had already been successful9, 10 . Also, it appeared possible that an absolute sensor could be developed whose response would depend on $1 y$ upon the strength of the field to be measured and the atomic constants of the gas molecules. Finally, from the considerable data avallable pertaining to the Stark 
effect in gases it was concluded that simple, reliable equipment could be used which would have few, if any, moving parts.

\section{MICROWAVE ABSORPTION IN GASES}

The theory of microwave absorption in gases has been developed in some detail11, 12, and the absorption has been shown to be closely connected with the rotational motions of the gas molecules. The rotational motion of a gas molecule depends upon its physical structure which is usually described in terms of the moments of inertia about three mutually perpendicular axes, $x, y$, and $z$, which pass through its center of mass. A molecule having equal moments of inertia about two of these axes, $x$ and $y$, is called a symmetric top, and this type of molecule was used in this method of field measurement. Only certain rotational energies are possible for a gas molecule, and the allowed states of rotation may be adequately described for the present application in terms of three integers or quantum numbers, $K, J$, and $M$. The quantum numbers describe the permitted angular momenta of the molecule. For example, the square of the molecule's tota 1 angular momentum, $P$, is given by

$$
\mathrm{P}^{2}=\frac{h^{2} J}{4 \pi^{2}}(J+1)
$$

where $h$ is Planck's constant. The projection, $P_{2}$, of the total angular momentum on the $\mathrm{z}_{z}$ axis of the molecule is given by

$$
P_{2}=\frac{h K}{2 \pi} \text {. }
$$

The projection, $P_{7}$, of the angular momentum on a polar axis, Z, fixed in space is

$$
P_{Z}=\frac{h M}{2 \pi} \text {. }
$$

Since the integers, $M$ and $K$, represent components of $J$, their magnitudes cannot exceed J. Therefore, for the symmetric top one may write

$$
\begin{aligned}
& J=0,1,2, \ldots \\
& \mathbf{K}=0, \pm 1, \pm 2, \pm 3, \ldots \pm \pm J \\
& M=0, \pm 1, \pm 2, \pm 3, \ldots \pm \pm J
\end{aligned}
$$

Absorption occurs in a gas whenever an $\mathrm{RF}$ electric field interacts with the dipole moment of a molecule and raises it from a given energy level to a higher one. When this occurs, the gas absorbs a photon whose frequency is given by the we11-known relation, $\nu=\frac{\Delta W}{h}$, where $V$ is the frequency, $h$ is Planck's constant, and $\Delta W$ is the energy difference between the two levels involved in the transition. The rotational energy of a symmetric top in a field-free region is

$$
W=\frac{P_{x}^{2}}{2 I}+\frac{P_{y}^{2}}{2 I}+\frac{P_{y}^{2}}{2 I} z
$$

where $I_{x}, I_{y}$, and $I_{z}$ are the moments of inertia about the $x, y$, and $z$ axes, respectively, and $P_{Y}, P_{y}$ and $P$ are the corresponding angular momenta. ${ }^{2}$ For the symmetric top, $I_{x}$ and $I_{y}$ are equal and each is called $\mathrm{I}_{\mathrm{y}^{\mathrm{x}}}^{\mathrm{x}}$ Equátion (5) may be
written

$$
W=\frac{p^{2}}{2 I_{y}}+P_{z}^{2}\left(\frac{1}{2 I_{z}}-\frac{1}{2 I_{y}}\right) \text {. }
$$

By expressing the momenta in terms of the quantum numbers one may write

$$
\frac{W}{h}=B J(J+1)+(C-B) K^{2}
$$

where $B=\frac{h}{8 \pi^{2} I_{y}}$ and $C=\frac{h}{8 \pi^{2} I_{z}}$. The

quantum number, $M$, does not enter into this expression because a spatial axis, $\mathrm{Z}$, is not established in this zero-field situation. All of the $(2 \mathrm{~J}+1)$ possible values of $M$ correspond to the same energy and 1 it is sald that an $M$ degeneracy of $(2 J+1)$ levels exists. The symmetry of the molecule requires that $\Delta K=0$ during an absorptive transition. This is because there is no electric dipole moment perpendicular to the $z$ axis of a symmetric top, and so no torque along this axis can be exerted by the RF electric field.

\section{THE STARK EFFECT}

The symmetric top has an electric dipole moment, $\mu$, along its axis of symmetry which possesses a component,

$\mu_{J}=\frac{\mu K}{V \sqrt{J(J+1)}}$ in the direction of angular momentum. The angle, $\alpha$, between the total angular momentum vector, $\mathrm{J}$, and a polar axis fixed in space is given by

$$
\cos \alpha=\frac{M}{\sqrt{J(J+1)}}
$$

If the axis in space is determined by an external dc electric field of magnitude, $E$, then there is a change in energy of the molecule caused by the interaction of its dipole moment with the field. This change is given by

$$
\Delta W_{J K M}=-\mu_{J} E \operatorname{Cos} \alpha=-\frac{\mu E K M}{J(J+1)} \text {. }
$$

From (7) and (9),

$$
\begin{aligned}
\frac{W_{J K M}}{h}=B J(J+1) & +(C-B) K^{2} \\
& -\frac{\mu E}{h} \frac{K M}{J(J+1)} .
\end{aligned}
$$


Thus the application of an electric field establishes a space orientation and removes the degeneracy of the energy levels. Transitions involving absorption may occur according to the rules,

$$
\begin{aligned}
& \Delta \mathrm{J}=+1 \\
& \Delta \mathrm{K}=0
\end{aligned}
$$

and $\Delta M=0, \pm 1$

If the static field is parallel to the RF electric field, then $\Delta M=0$, since the $\mathrm{RF}$ field cannot produce torque along the $Z$ axis. If the static and $R F$ electric fields are perpendicular 13 , then $\Delta M= \pm 1$. For a transition from $J=J$ to $\mathrm{J}=\mathrm{J}+1$, and for $\Delta \mathrm{M}=0$, the frequency of absorption is found from (10) to be

$$
\begin{aligned}
V & =\frac{W_{(J+1) K M}}{h}-\frac{W_{J K M}}{h}=2 B(J+1)+ \\
& +\frac{2 \mu E}{h} \frac{K M}{J(J+1)(J+2)} .
\end{aligned}
$$

For $\Delta M= \pm 1$ it is

$$
\begin{aligned}
V & =\frac{W_{(J+1) K(M \pm 1)}}{h}-\frac{W_{J K M}}{h}=2 B(J+1) \\
& +\frac{(2 M \mp J) K \mu E}{h J(J+1)(J+2)}
\end{aligned}
$$

The frequency shift is a linear function of the static field in both cases. For $\Delta M=0$ the shift is

$$
\Delta V=\frac{2 \mu \mathrm{KME}}{\mathrm{hJ}(\mathrm{J}+1)(\mathrm{J}+2)} \text {. }
$$

For $\mathcal{V}$ in megahertz, $\mu$ in Dybye units, and F in volts per centimeter, the quantity, , is equal to 1.0064 . For $\mu=1$ Debye unit, the maximum shift occurs when $K=M=J$, and it is given by

$$
\Delta V \approx \frac{\mathrm{JE}}{(\mathrm{J}+1)(\mathrm{J}+2)} \text { megahertz. }
$$

For a typical case of $J=3$ the frequency shift is

$$
\Delta \downarrow \approx 0.15 \mathrm{E} \text { megahertz. }
$$

It is interesting at this point to compare (16) with results obtained from tests where energy levels in magnetic materials were shifted by the application of dc magnetic fields. In one case14 a shift of 2.8 megahertz per gauss was used to obtain a measure of the dc magnetic field to values as small as $10^{-7}$ gauss. If equal precision could be achieved in measuring frequency shifts in rotational spectra, equation (16) indicates that one could expect to measure electric fields the order of one millivolt per meter by means of the Stark effect.
THE AMMONIA ABSORPTION LINE

Ammonia was chosen as the first gas to be used in this application because it has a very strong absorption line in its microwave spectrum which exhibits a frequency shift in the presence of a dc electric field. The equation of the absorption line in the pressure range of interest is 15

$$
\gamma=k_{1} \frac{\Delta}{\left(\nu-\nu_{0}\right)^{2}+\Delta^{2}}
$$

where $\gamma$ is the absorption coefficient at the frequency, $V$, and is expressed in $\mathrm{cm}^{-1}$. $\mathrm{K}_{1}$ is a constant of proportionality. The frequency difference between the two half-power points of the line is $2 \Delta$, and $\mathcal{V}$ is the resonant frequency, or frequency of maximum absorption. Frequencies are expressed in megahertz. The expression, (17). may be written as

$$
\gamma=\frac{k_{2}}{u_{k_{1}}^{2}+1}
$$

where $\mathrm{K}_{2}=\frac{\mathrm{u}_{\mathrm{K}}+1}{\Delta}$ and $\mathrm{u}=\frac{\nu-\nu_{\mathrm{O}}}{\Delta \Delta}$. The variable, $\{$, is the frequency deviation from resonance expressed in units of $\Delta$.

Ammonia is a pyramid-shaped molecule with the nitrogen atom at the apex and the three hydrogen atoms in the same plane and equidistant from the nitrogen atom. The nitrogen vibrates along the symmetry axis of the molecule and often passes through the plane of the hydrogen atoms and resumes its vibrational motion on the other side of the molecule16. Because of this shuttling action, the molecule in a field-free region has no average dipole moment, although it has a permanent dipole moment, $\mu$, in either stable physical configuration. The average dipole moment is a function of the applied electric field, and so the energy of orientation is a non-linear function of this external field. The shift in frequency is a second order effect and is reported to be 17

$$
\begin{aligned}
\Delta V= & 0.5065 \frac{\mu^{2} E^{2}}{V_{0}} \times \\
& \times\left[\frac{\mathrm{KM}}{J(J+1)}\right]^{2} \text { megahertz }
\end{aligned}
$$

where $\mu$ is in Debye units, $E$ is in volts per centimeter, and $\Delta M=0 . V$ is the resonant frequency of the unshifled line. For the line used in this work, $J=K=3$, and $M$ may be $0, \pm 1, \pm 2$, or \pm 3 . From (19) it is seen that the amount of shift for a particular molecule depends upon the value of $M$ describing its state. Therefore, the absorption frequency of some molecules is shifted more than others and the overall absorption line shape is changed from that described in equation (17). The behavior of this ammonia line 
in a dc electric field is indicated in Figure 1.

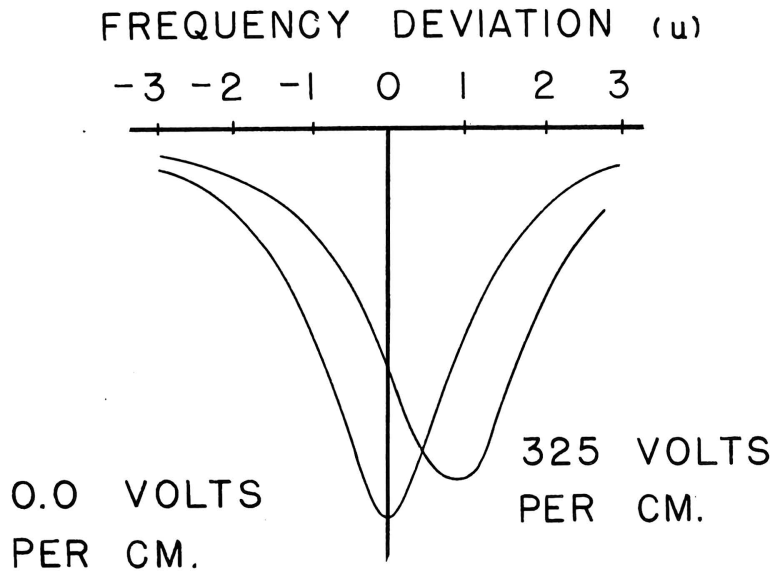

Figure 1. The Ammonia Absorption Line For Two Values of Electric Field.

THE EXPERIMENTAL SYSTEM

The simple microwave system shown in Figure 2 was constructed to demonstrate

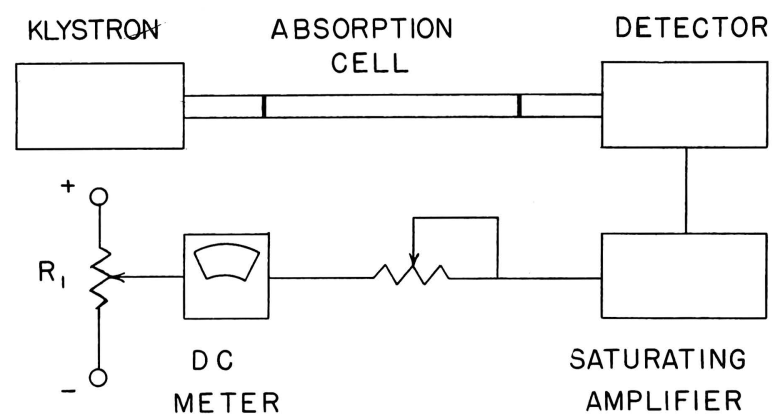

Figure 2. The Microwave System Used To Obtain a Direct Indication of the Applied Electric Field. the use of the Stark effect in electric field instrumentation. It was not designed to perform precise measurements, but merely to provide some direct indication of the approximate magnitude of an applied field, and thereby justify this approach. Microwave energy at a frequency of approximately $2.4 \times 10^{10}$ hertz was propagated through the absorption cell to the diode detector. When the klystron frequency was swept in synchronism with the oscilloscope trace, the absorption line shown in Figure 3 was observed on the oscilloscope. The dc electric field was established by means of an electrode within the absorption cel1.

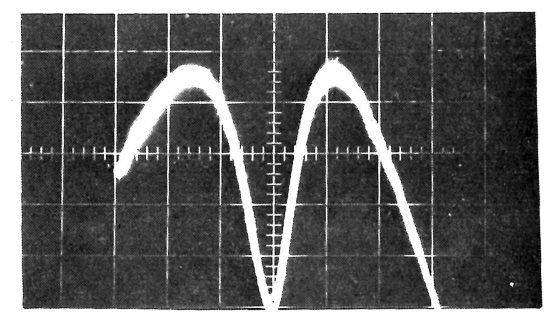

$E=0.0$ VOLTS PER CM. (A)

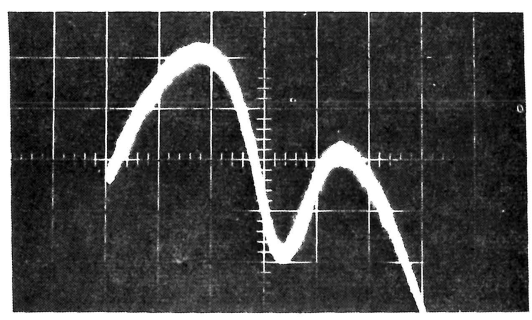

$E=140$ VOLTS PER CM.

(B)

Figure 3. The Ammonia Absorption Line Superimposed Upon The Operating Mode of the Klystron for Two Values of Electric Field.

The detection system of Figure 2 was arranged to indicate a change in width of periodic pulses coming from the detector. The high-gain saturating amplifier provided a rectangular output pulse of constant amplitude whose width was a function of the width of the pulse from the detector. The dc meter provided a measure of the average value of the amplified signal. $R_{1}$ was adjusted so that the meter reading was zero when the electric field applied to the ammonia was zero.

In order to obtain a pulse which exhibited an appreciable change in width when a 
dc field was applied to the absorption ce11, the modulation scheme of Figure 4 was used. With this method, the frequency of the klystron oscillator was set at some value, $V_{1}$, which was near to but

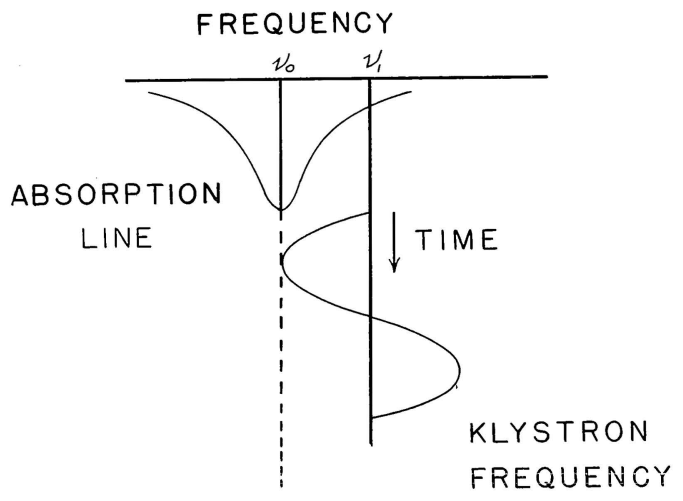

Figure 4. Klystron Frequency as a Function of Time.

higher than $\mathcal{V}$. The frequency was then varied sinusoidlily so as to sweep one half of the absorption line twice each cycle. When the electric field modified the absorption line by changing its resonant frequency, its width, and its amplitude, the oscillator frequency swept more than one half of the line twice during each cycle. The detector output pulse then experienced a decrease in amplitude and a broadening which was caused both by line broadening and by the resonant frequency shift. This is shown in Figures 5 and 6.

A vacuum tube voltmeter was used to measure the output of the system. A number of factors affected the shape of the diode detector's output pulse, and, therefore affected the signal to the meter. The observed absorption line shape was distorted to some extent by the mode shape of the klystron and also by a somewhat uneven electric field distribution within the absorption cell. Also, reflections within the microwave system as well as the response of the electronic circuitry affected the output pulse shape. The

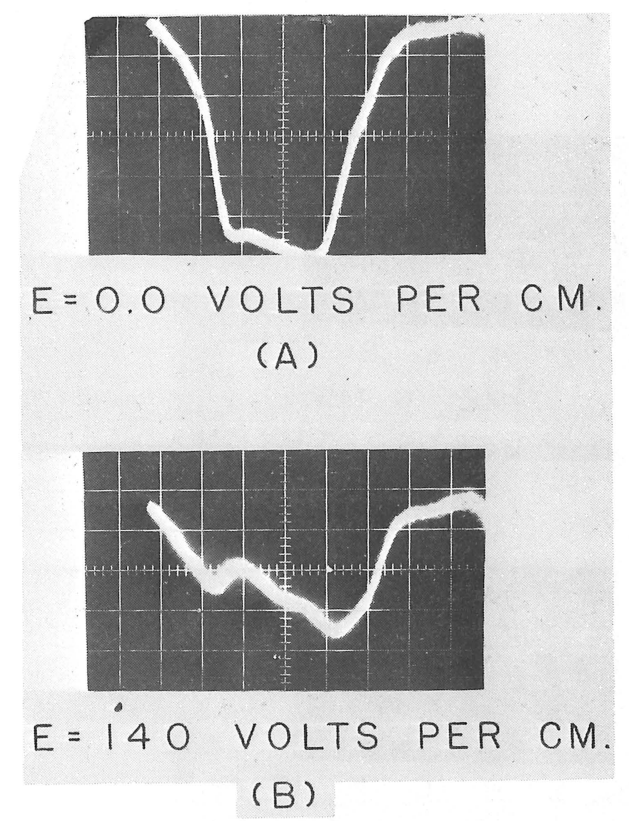

Figure 5. Detector Output of the Modulated System for Two Values of Electric Field.

observed system output as a function of the applied electric field was fitted with the computed response at the two extreme points and plotted in Figure 7 to obtain a comparison of predicted and observed results. The minimum detectable electric field in this system was estimated to be approximately fifty volts per centimeter.

\section{CONCLUSIONS}

A1though this system has not been refined to the point where its ultimate sensitivity can be determined, it has demonstrated that the Stark effect can be used to measure static electric fields. Work is continuing in an effort to develop a practical stark effect field meter. A number of significant points are being considered:

1. Arrangements are being made to measure the field external to the equipment rather than the dc field within the absorption cell as described in this paper.

2. The effect of the orientation of the instrument will be examined. In this work the dc field was always parallel to the RF electric field, a condition which resulted in the selection rule, $\Delta M=0$. 


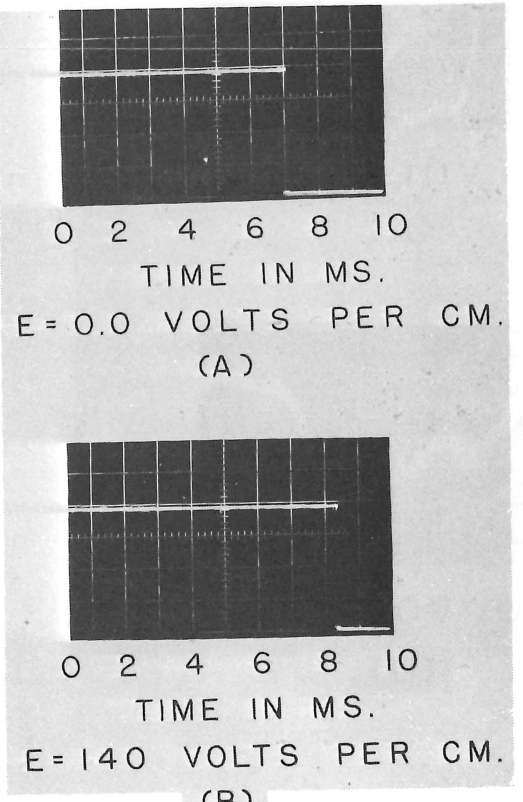

(B) $\odot$ OBSERVED
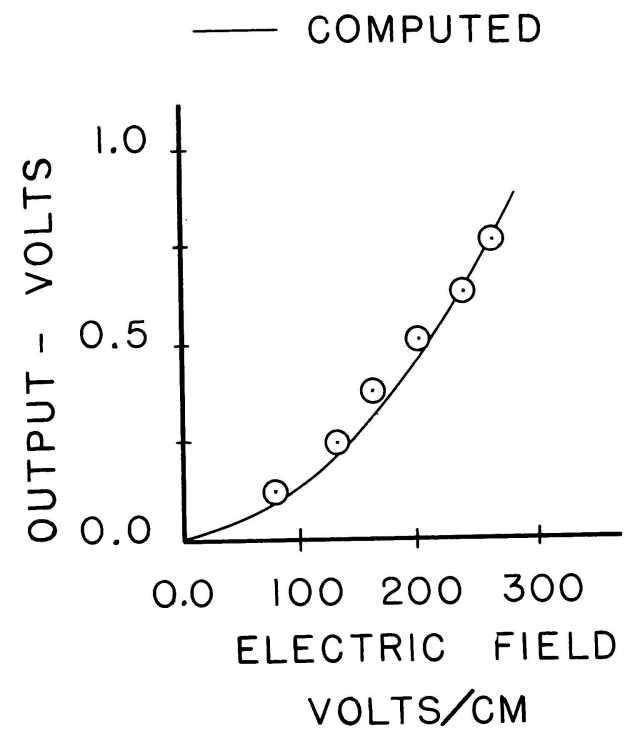

Figure 7. Output Voltage as a Function of the Electric Field.

2. G. W. Paltridge, "Measurement of the Electrostatic Field in the Stratosphere," J. GEOPHYS. RES., vo1. 69, pp. 1947-1954; May, 1964.

3. W. W. Mapleson and W. S. Whitlock, "Apparatus for the Accurate and Continuous Measurement of the Earth's Electric Field," J. ATMOS. TERR. PHYS., vol. 7, pp. 61-72; 1955.

4. G. L. Gdalevich, "Rocket Experiments Aimed at Detecting an Electric Field in the Ionosphere," in "Problems of Atmospheric and Space Electricity", Elsevier Publishing Co., pp. 566-572; 1965. 5. J. L. Wildman, "A Device for Measuring Electric Field in the Presence of Ionisation," J. ATMOS. TERR. PHYS., vo1. 27, p. 417; March 1965.

6. J. M. Palmer, "A New Electrostatic Field Measuring Instrument," IEEE TRANS. ON AEROSPACE, vol. AS-3, pp. 523-527; June 1965.

7. D. W. Johnson and A. Kavadas, "A Rocket-Borne Electric Field Probe," CANADIAN

J. PHYS., vol. 41, pp. 1980-1990; 1963.

8. R. Munoz, "The Ames Magnetometer,"

NAT. TELEMETERING CONF. PROC., pp. 77-80, Boston, Mass.; 1966.

9. L. D. Schearer, F. D. Colegrove, and G. K. Walters, "Optically Pumped Nuclear Magnetometer," REV. SCI. INSTR., vol. 34, No. 12, p. 1363; December, 1963.

10. A. R. Keyser, J. A. Rice, and L. D.
1. J. Alan Chalmers, "Atmospheric Electricity", Macmillan Co., New York, N. Y; 1957 .

\section{REFERENCES}


Schearer, "A Metastable Helium Magnetometer for Observing small Geomagnetic Fluctuations," J. GEOPHYS. RES., vol. 66, No. 12, 4163; December 1961.

11. B. Bak, "Elementary Introduction to Molecular Spectra," Interscience Publishers, Inc., New York; 1962.

12. C. H. Townes and A. L. Schawlow, "Microwave Spectroscopy," McGraw-Hill Book Company, Inc., New York, N. Y., ch. 1; 1955.
13. Townes and Schawlow, Ibid., p. 235.

14. Keyser, Rice, and Schearer, loc. cit. 15. Townes and Schawlow, op. cit., p. 343.

16. E. Merzbacher, "Quantum Mechanics," John Wiley and Sons, Inc., New York, N. Y., pp. 64-77; 1961 .

17. W. Gordy, W. V. Smith, and R. F. Trambarulo, "Microwave Spectroscopy," John Wiley and Sons, Inc., New York, N. Y., P. 158 ; 1953. 



\section{PUBLICATIONS OF THE ENGINEERING REPRINT SERIES}

Copies of publications may be secured from the Director of the Engineering Experiment Station, University of Missouri. Single copies may be obtained free unless otherwise indicated until the supply is exhausted. Requests for additional copies will be considered upon further inquiry.

Reprint No.

68. A Method of Data List Processing with Application to EEG Analysis by C. M. Philpott, Control Data Corporation, St. Paul, Minnesota, and G. B. Lago, Professor of Electrical Engineering, University of Missouri. Reprinted from Communications of the ACM, Volume 8, Number 5, May, 1965.

69. Method for Obtaining the Trees of a v Vertex Complete Graph from the Trees of a v-1 Vertex Complete Graph by G. W. Zobrist, Assistant Professor of Electrical Engineering, University of Missouri, and G. V. Lago, Professor of Electrical Engineering, University of Missouri. Reprinted from the Matrix and Tensor Quarterly, Volume 15, Number 3, March, 1965.

70. Treatment of Livestock Waste-A Laboratory Study by E. A. Jeffrey, W. C. Blackmann, Jr., and Ralph Ricketts. Reprinted from Transactions of the ASAE, Volume 8, Number 1.

71. The Electronic Position Indicator by Richard P. Covert, Associate Professor of Industrial Engineering, University of Missouri. Reprinted from The Journal of Industrial Engineering, Volume XVI, No. 4, July-August, 1965, pages 255-259.

72. The Reflected Impedance of a Circular Coil in the Proximity of a Semi-Infinite Medium by David H. S. Cheng. Reprinted from IEEE Transactions on Instrumentation and Measurement, Volume IM-14, Number 3, September, 1965.

73. Irrotational Flow Over Spillways of Finite Height by John J. Cassidy, Associate Professor of Civil Engineering, University of Miss ourị. Reprinted from Journal of the Engineering Mechanics Division, Proceedings of the American Society of Civil Engineers, Volume 91, Number EM6, December, 1965.

74. Experimental Investigation of a Magnetically Balanced Arc in a Transverse Argon Flow by T. W. Myers, Visiting Research Associate, Thermo-Mechanics Research Laboratory, Wright-Patterson AFB, Ohio, C. N. McKinnon, Instructor of Mechanical Engineering, University of Missouri, and J. C. Lysen, Associate Professor of Mechanical Engineering, University of Missouri. Reprinted from Journal of Engineering for Power, January, 1966.

75. A Study of the Effects of Paced Audio-Rhythm on Repetitive Motion by John A. Conte, Undergraduate Industrial Engineer, University of Missouri. Reprinted from The Journal of Industrial Engineering, Volume XVII, Number 3, 1966.

76. Measured and Computed Stresses in Three Castellated Beams by Richard T. Douty, Associate Professor of Civil Engineering and James W. Baldwin, Professor of Civil Engineering, University of Missouri. Reprinted from AISC Journal, January, 1966.

77. A Method of Obtaining a Uniform Electric Field by A. V. Dralle and D. L. Waidelich. Reprinted from 1966 IEEE International Convention Record, Part 7.

78. The Use of the Flux Plot in Traffic Control by Robert J. Wheeler, Associate Professor of Civil Engineering and Elmer M. Tory, Associate Professor Mathematics, Mount Allison University. Reprinted from the Traffic Quarterly, July 1965.

79. A Statistical Definition of Perfect Mixtures of Solids of Different Sizes by Kun Sup Hyun, Graduate Student in Chemical Engineering, and L. E. Marc De Chazal, Professor of Nuclear and Chemical Engineering, University of Missouri. Reprinted from I \& EC Process Design and Development, Vol. 5, April 1966.

80. A Qualitative and Quantitative Measure of Aufwuchs Production, by Darrell L. King, Assistant Professor of Civil Engineering, University of Missouri and Robert C. Ball, Professor of Fisheries and Wildlife, Michigan State University. Reprinted from Trans. Amer. Micros. Soc. $85(2): 232-240.1966$.

81. Hetergeneous Catalysis: Effect of an Alternating Electric Field by Vin-Jang Lee, Assistant Professor of Chemical Engineering, University of Missouri. Reprinted from Science, April 22, 1966, Vol. 152, No. 3721, page 514.

82. Methods of Measuning Electric Fields by Byron W. Sherman, Assistant Professor of Electrical Engineering, University of Missouri and Donald L. Waidelich, Professor of Electrical Engineering, University of Missouri. Reprinted from Supplement to IEEE Transactions on Aerospace and Electronic Systems, Vol. AES-2, No. 6, Nov. 1966. 



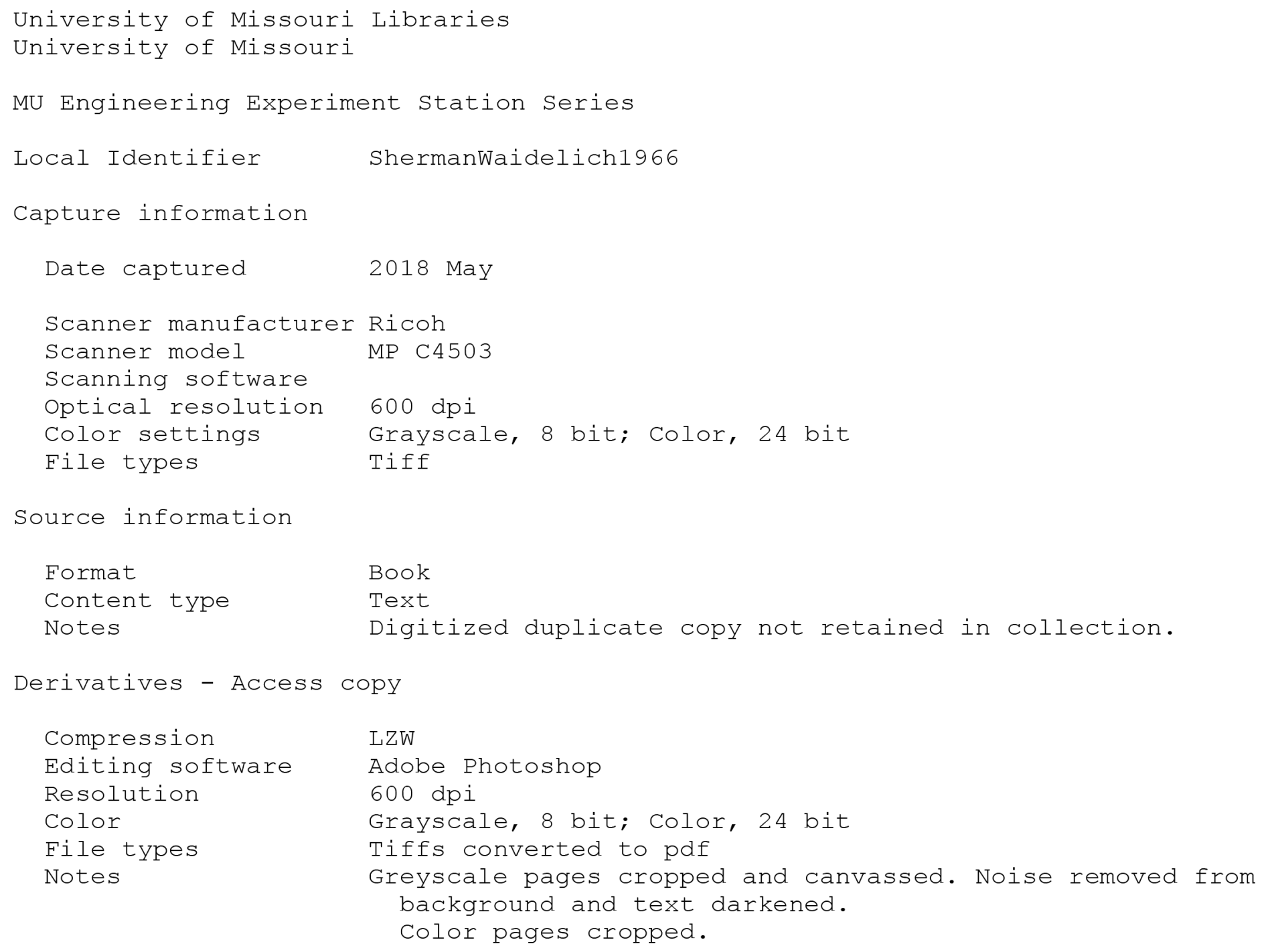

